\title{
FEICA European Adhesives \& Sealants Conference and EXPO 2013
}

FEICA (Association of the European Adhesive \& Sealant Industry) invites all those interested in adhesives and sealants to attend the FEICA European Adhesives \& Sealants Conference, which will be held on September 11-13 2013 in Izmir, Turkey.

T he FEICA annual conference is a jour fixe for the adhesive and sealant industry in Europe. Started in 1972 , the aim of the conference is to give a platform to promote discussion and cooperation between multinational and SME formulators and raw material suppliers in the adhesive \& sealant industry. It is a unique opportunity to meet peers, suppliers and others with an interest in the industry, and an ideal setting to discuss common business, technical and regulatory issues and gain an inside view of current market and innovation opportunities.

The conference program brings together Europe's leading business thinkers and experts to share their experience and knowledge and to equip participants with the tools they need to meet current and future challenges.

This year's Business Forum starts on Thursday, September 12 under the theme "Adapt and change, the Key to
Growth in Europe". The first keynote speaker, Swedish Futurologist Magnus Lindkvist, highlights trends in consumption, technology, consumer behavior and strategy and combines quantitative business factors with future visionary art. "Business is all about focus", says Magnus. "But with focus comes myopia and to conquer that, I come in to shake things up. To be invited as a keynote speaker is to have a license to interfere and interrupt."

The second keynote speaker is the well-known economist Roger MartinFagg, who teaches applied economics and strategy for Executive and Corporate programs. Roger has extensive knowledge of the manufacturing, distribution, financial services and energy sectors. He also earned a reputation for turning complex economic issues into clear, concise and practical interpretations and has been described as one of the few economists with a keen sense of humor.

Furthermore, breakout sessions on both conference days address the following topics:

- Sustainable Development - a $360^{\circ}$ approach

- Innovations in Hot Melt Technology

- Solutions for Small \& Medium Sized Companies - Turning Challenges into Opportunities

- Smart Innovation in Adhesives \& Sealants

- Business and Hot Melt

- Advances in Dispersion Adhesive Technology

- Spotlight on Turkey

The tabletop Exhibition, a buzzing hub at the centre of the conference, runs on September 12 and 13 parallel to the lectures and gives the attendees the opportunity to exchange information on the latest developments in the adhesive and sealant industry.

For more information, please visit www.feica-conferences.com

\section{Delo Founds Subsidiary in China}

After having founded a subsidiary in Singapore at the end of 2012, Delo Industrie Klebstoffe has now established a further company base in the Asian region: Delo Industrial Adhesives (Shanghai), Co. Ltd.

C hina is a key sales market for Delo. Its long-term trading partner is Electronic Scientific Engineering Ltd. (ESE) located in Hong Kong. "Thanks to the proven collaboration with our Chinese partner ESE, we have been successful there for more than 10 years. We are pleased to have Shanghai as our second subsidiary in Asia”, said Sabine Herold, Managing Partner of the company.

In the future, the company will have warehouse capacities for adhesives and will provide service for devices such as LED curing lamps. "In this way, we can satisfy our Chinese customers' wishes and needs even better", Sabine Herold added. Delo operates globally and is represented by its own employees in 12 countries on three continents. The company generates around one third of its sales revenues in Asia.

Delo has now founded a second subsidiary in Asia.

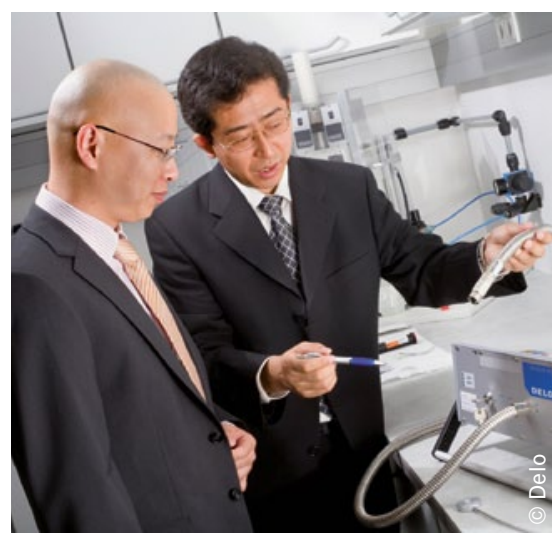

